\title{
Primary cardiac angiosarcoma directly invading the right lung: An autopsy report
}

\author{
Takumi Kiwaki ${ }^{1}$, Akira Ishihara ${ }^{2}$, Takanori Toyama ${ }^{2}$, Hiroaki Kusaka ${ }^{2}$, and Hiroaki \\ Kataoka $^{1}$ \\ ${ }^{1}$ University of Miyazaki \\ ${ }^{2}$ Miyazaki Prefectural Nobeoka Hospital
}

October 22, 2020

\begin{abstract}
A 60-year-old man presented to our hospital with pericardial effusion. He died of respiratory failure 13 months later. Autopsy revealed right atrial angiosarcoma extending into the right lung. To the best of our knowledge, this is the first case report of a primary cardiac angiosarcoma that displayed direct lung invasion.

\section{Primary cardiac angiosarcoma directly invading the right lung: An autopsy report}

Takumi Kiwaki $(\mathrm{MD})^{1^{*}}$, Akira Ishihara $(\mathrm{MD}, \mathrm{PhD})^{2,3}$, Takanori Toyama $(\mathrm{MD})^{4}$, Hiroaki Kusaka (MD, $\mathrm{PhD})^{5}$ and Hiroaki Kataoka $(\mathrm{MD}, \mathrm{PhD})^{1}$

${ }^{1}$ Section of Oncopathology and Regenerative Biology, Department of Pathology, Faculty of Medicine, University of Miyazaki, Miyazaki, Japan

${ }^{2}$ Department of Pathology, Miyazaki Prefectural Nobeoka Hospital, Miyazaki, Japan

${ }^{3}$ Department of Pathology, Junwakai Memorial Hospital, Miyazaki, Japan

${ }^{4}$ Department of Internal Medicine, Miyazaki Prefectural Nobeoka Hospital, Miyazaki, Japan

${ }^{5}$ Department of Cardiovascular Medicine, Miyazaki Prefectural Nobeoka Hospital, Miyazaki, Japan

*Correspondence: Takumi Kiwaki

Section of Oncopathology and Regenerative Biology, Department of Pathology, Faculty of Medicine, University of Miyazaki, 5200, Kihara, Kiyotake, Miyazaki, 889-1692, Japan.

E-mail:takumi_kiwaki@med.miyazaki-u.ac.jp

Tel: +81-985-852809, Fax: +81-985-856003
\end{abstract}

\section{Keywords}

Primary cardiac angiosarcoma, Angiosarcoma, Cardiac tumor, Cardiac malignancy, Autopsy

\section{Key Clinical Message}

A 60-year-old man presented to our hospital with pericardial effusion. He died of respiratory failure 13 months later. Autopsy revealed right atrial angiosarcoma extending into the right lung. To the best of our knowledge, this is the first case report of a primary cardiac angiosarcoma that displayed direct lung invasion.

\section{Introduction}


Tumors of the heart are rare, the majority of which are metastatic tumors ${ }^{1}$. Primary cardiac tumors are extremely rare, accounting for only $0.0017 \%-0.19 \%$ of unselected patients at autopsy ${ }^{2}$. Seventy-five percent of them are benign neoplasms and the remainders malignant. Myxomas are by far the most common benign cardiac tumors ${ }^{3}$. As for malignant tumors arising primarily in the heart, angiosarcomas are one of the most common types ${ }^{4,5}$. Primary cardiac angiosarcoma (PCA) originates in vascular endothelial cells and commonly arises in the right atrial wall ${ }^{5,6}$. It exhibits a poor prognosis despite surgical treatment, chemotherapy, and radiotherapy ${ }^{1}$. Here, we present a case of PCA that was diagnosed for the first time at autopsy.

\section{Case report}

A 60-year-old previously healthy Japanese man was referred to our hospital for evaluation of anemia and pericardial effusion 13 months before his death. The patient presented with no apparent symptoms at that time. Pericardiocentesis was performed, and the effusion was evaluated cytologically; however, no atypical cells could be identified, and the etiology of the effusion was undefined. The patient ignored follow-up visit appointments; but returned six months later to the hospital with dyspnea, bloody sputum, edema, and anorexia. A blood examination showed severe anemia (red blood cells, $1.64 \times 10^{6} / \mu \mathrm{L}$; hemoglobin, $3.3 \mathrm{~g} / \mathrm{dL}$ ), and echocardiography revealed a mass in the right atrium (Figure 1). Computed tomography (CT) disclosed multiple nodules in the lungs and the liver. As a biopsy of the cardiac mass was technically difficult, a transbronchial biopsy of the lung mass was performed; but the biopsied specimen did not contain any atypical cells. Hematological malignancies were suspected because of progressive anemia and the presence of human T-cell leukemia virus type 1 antibodies. The patient was treated with THP-COP (pirarubicin, cyclophosphamide, vincristine, and prednisolone) and mogamulizumab under the provisional diagnosis of adult T-cell leukemia/lymphoma. Nevertheless, the patient's general condition worsened progressively. The anemia and edema continued to progress, and the pleural effusion increased. The patient died of respiratory failure 13 months after his initial admission to the hospital.

The patient was autopsied eight hours after his death. The heart adhered to the pericardium (Figure 2A), and the pericardial cavity contained $130 \mathrm{~mL}$ of sanguineous pericardial effusion. In the right atrium, a dark-red colored, approximately $7 \times 4$-cm-sized multilobulated tumor arose from the atrial wall and filled the dilated atrial lumen (Figure 2B). The cut surface of the tumor was sanguineous and solid, intermingled with necrotized and calcified foci (Figure 2C). The muscular layer of the right atrial wall was completely replaced by the tumor. The tumor penetrated the pericardium and directly invaded the right lung (Figure 2D). Histologically, the tumor consisted of atypical cells with oval- to spindle-shaped nuclei, which formed vague capillary channels containing red blood cells (Figure 3A). Tumor cells protruded into the atrial cavity, forming papillary structures (Figure 3B). Exfoliated clusters of tumor cells were also observed in the atrium. The tumor cells were immunoreactive for CD31 (Figure 3C) and FLI1 but were negative for podoplanin (D2-40 antibody). Therefore, we diagnosed the lesion as an angiosarcoma. The tumor cells penetrated the pericardium and visceral pleura of the right lung and directly invaded the pulmonary parenchyma. Multiple metastases were identified in the lungs, liver, spleen, jejunum, and skin. In the jejunum, the metastatic nodule resulted in intussusception. In the bilateral lungs, tumor emboli were formed in the pulmonary arteries, and intra-alveolar hemorrhage and bronchopneumonia were present. The central nervous system was not examined.

\section{Discussion}

In this case, the patient did not present with symptoms at the first visit. The symptoms of PCA are usually nonspecific and include chest pain, shortness of breath, malaise, and fever ${ }^{1}$. Pericardial effusion is also common in patients with PCA; however, in general, the detection of angiosarcoma cells is difficult even with a cytological evaluation of the pericardial fluid ${ }^{7}$, as seen in our patient. Hence, when one encounters a patient with unexplained pericardial effusion, the possibility of malignancies including PCA should not be excluded, even with a "negative" result of the pericardial effusion cytology.

Given the low frequency of PCA and a relatively higher incidence of metastatic cardiac tumor, diagnosis of 
cardiac angiosarcoma as the primary lesion is sometimes challenging. In the current case, we encountered this difficulty as the patient had multiple tumor nodules in the liver, spleen, and skin. Besides the heart, angiosarcomas can arise in the soft tissue, breast, liver, bone, spleen, or other visceral organs ${ }^{8}$. Furthermore, the incidence of angiosarcoma in the cutaneous soft tissue, liver, or spleen is higher than that in the heart ${ }^{8}$. In this regard, Cunha-Silva et. al.suggested the following factors to evaluate whether the cardiac angiosarcoma is primary or not ${ }^{9}$ : (1) patient's age and lifestyle history, (2) number of lesions (i.e., single or multiple) in the heart, and (3) location of the lesion in the heart. In addition, while cardiac metastasis of liver angiosarcoma is rare, the liver is a common metastatic site of $\mathrm{PCA}^{9}$. In the current case, the patient had no history of exposure to thorium oxide, vinyl chloride, arsenic, or anabolic steroids, all of which are risk factors for hepatic angiosarcoma. Moreover, the cardiac lesion was single, and the hepatic lesions were multiple. The cardiac lesion located in the right atrium, the most common site for PCA. These findings strongly suggest that the cardiac angiosarcoma in this case was PCA.

Pericardial extension of the tumor was observed in $71 \%$ of 17 cases of $\mathrm{PCA}^{7}$, and cardiac rupture due to PCA has also been reported ${ }^{10}$. However, to the best of our knowledge, a case of PCA with direct lung invasion has not been described previously. In the current case, the tumor penetrated the pericardium and invaded the lung. However, neither fatal cardiac tamponade nor rupture occurred in this case. Although the incidence of lung invasion may be low, it may be meaningful for the clinical management of PCA to recognize the possibility of lung invasion.

In conclusion, we presented an autopsy case of PCA that directly invaded the right lung. Although PCA is an extremely rare disease, it should be included in the differential diagnosis of a cardiac mass with pericardial effusion.

\section{Acknowledgement}

None.

\section{Conflict of interest}

The authors have no conflicts of interest to disclose.

\section{Author contributions}

TK: performed autopsy and drafted the manuscript, AI: performed autopsy and edited the manuscript, TT: treated the patient and reviewed the manuscript, H Kusaka: treated the patient and reviewed the manuscript, H Kataoka: supervised autopsy diagnosis and manuscript preparation.

\section{References}

1. Butany J, Nair V, Naseemuddin A, Nair GM, Catton C, Yau T. Cardiac tumours: diagnosis and management. Lancet Oncol. 2005;6:219-28.

2. Reynen K. Cardiac myxomas. N Engl J Med. 1995;333:1610-7.

3. Silverman NA. Primary cardiac tumors. Ann Surg.1980;191:127-38.

4. Chen TW, Loong HH, Srikanthan A, Zer A, Barua R, Butany J, Cusimano R, Liang Y, Chang C, Iakobishvili Z, Razak AA, Lewin J. Primary cardiac sarcomas: A multi-national retrospective review. Cancer Med.2018;8:104-10.

5. Neuville A, Collin F, Bruneval P, Parrens M, Thivolet F, Gomez-Brouchet A, Terrier P, Montpreville VT, Gall FL, Hostein I, Lagarde P, Chibon F, Coindre J. Intimal sarcoma is the most frequent primary cardiac sarcoma: Clinicopathologic and molecular retrospective analysis of 100 primary cardiac sarcomas. Am J Surg Pathol . 2014;38:461-9.

6. Leduc C, Jenkins SM, Sukov WR, Rustin JG, Maleszewski JJ. Cardiac angiosarcoma: histopathologic, immunohistochemical, and cytogenetic analysis of 10 cases. Hum Pathol. 2017;60:199-207.

7. Kupsky DF, Newman DB, Kumar G, Maleszewski JJ, Edwards WD, Klarich KW. Echocardiographic features of cardiac angiosarcomas: The Mayo Clinic experience (1976-2013). Echocardiogr Mt Kisco N.2016;33:186-92. 
8. Young RJ, Brown NJ, Reed MW, Hughes D, Woll PJ. Angiosarcoma.Lancet Oncol . 2010;11:983-91.

9. Cunha-Silva M, Mazo DF de C, Gomes ILC, Imbrizi MR, Seva-Pereira T, Vaz NF, Lourenco MS, Costa LBF, Escanhoela CAF, Almeida JRS. Multiple hepatic metastases of cardiac angiosarcoma. Autopsy Case Rep . 2018;8:e2018048

10. Kim J, Da Nam B, Hwang JH, Park SB, Lee M, Kim DW, Park YW, Lee M. Primary cardiac angiosarcoma with right atrial wall rupture.Medicine (Baltimore) . 2019;98:e15020

\section{Figure Legends}

\section{Figure 1}

Transesophageal echocardiogram of the heart tumor. A mass was observed in the right atrium. A papillary structure was also discovered (yellow arrowhead). T, tumor; RA, right atrium; RV, right ventricle.

\section{Figure 2}

Macroscopic examination of the autopsy. (A) The heart adhered to the pericardium. The right atrial tumor was connected to the pericardium (yellow asterisk). RA, right atrium; RL, right lung; P, pericardium. (B) A dark-red colored tumor was observed in the right atrium. The specimen was cut along the yellow line. (C) Cut surface of the heart tumor. (D) Cut surface of the right lung. The tumor invaded the pleura and the lung parenchyma. The visceral pleura was penetrated by the tumor cells in the yellow arrowhead portion. Multiple metastases were also identified. Portions indicated by yellow asterisks of Figure $2 \mathrm{C}$ and $2 \mathrm{D}$ are connected to each other, and the portions correspond to the yellow asterisks in Figure 2A. Bars, $1 \mathrm{~cm}$

\section{Figure 3}

Histological and immunohistochemical findings of the autopsy specimen. (A) and (B) H\&E staining of the heart tumor. (A) Atypical tumor cells with vascular formation were observed. Bar, $50 \mu \mathrm{m}$ (B) The tumor cells with a papillary structure protruded into the atrial cavity. Bar, $1 \mathrm{~mm}$ (C) CD31 immunostaining revealed vascular differentiation of the tumor cells. Bar, $100 \mu \mathrm{m}$

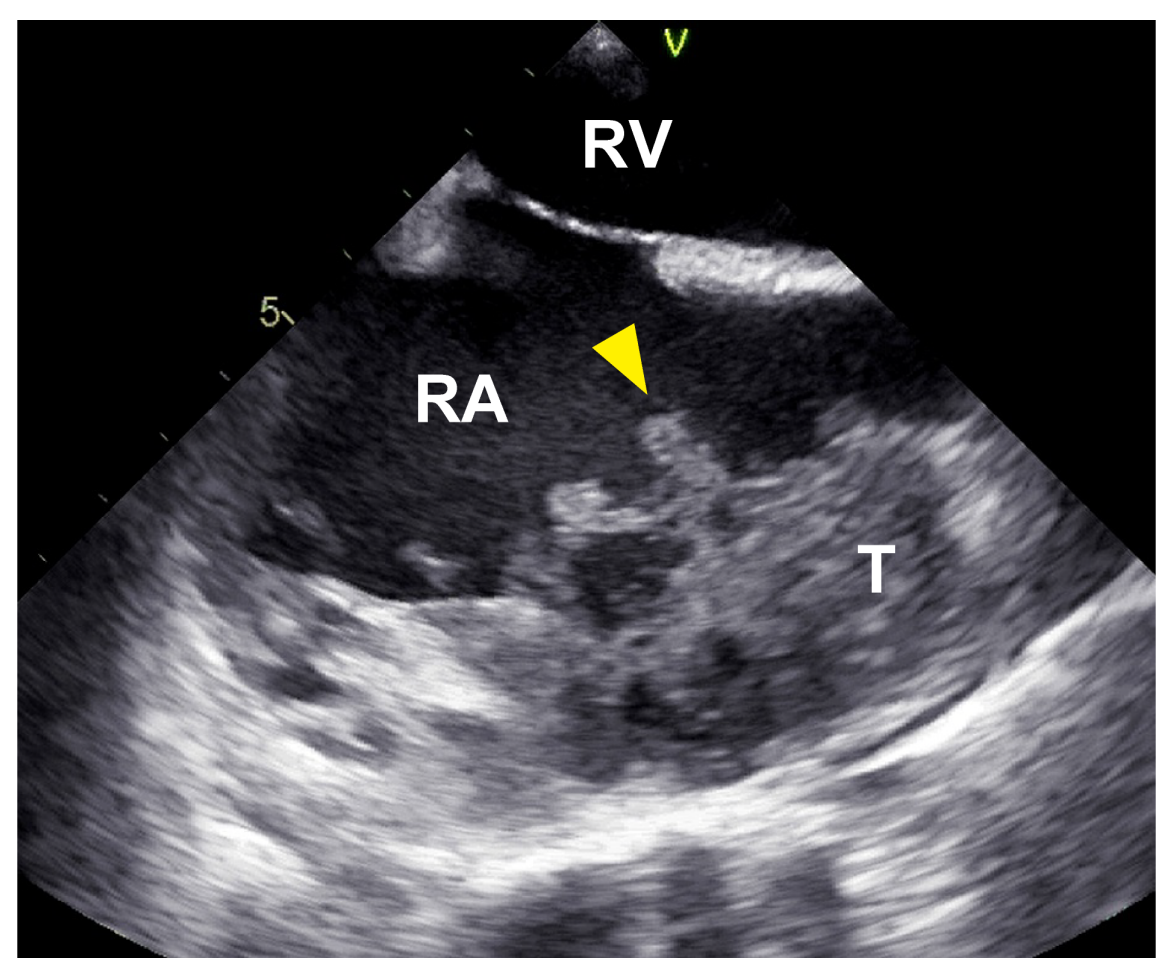



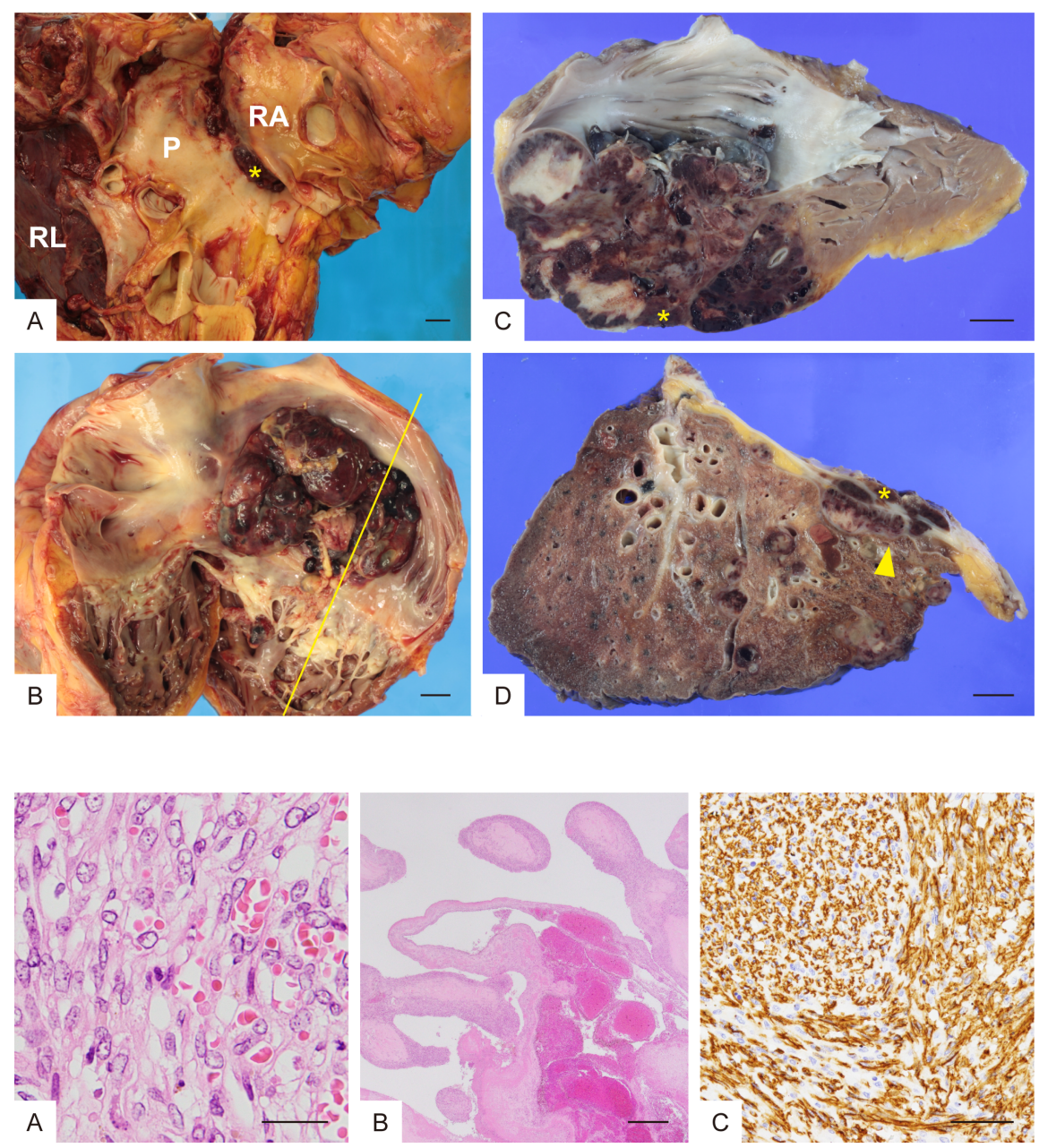